\title{
A Pictorial Technique for Mass Screening of Sorghum Germplasm for Anthracnose (Colletotrichum sublineolum) Resistance
}

\author{
Louis K. Prom ${ }^{*}, 1$, Ramasamy Perumal ${ }^{2}$, John Erpelding ${ }^{3}$, Thomas Isakeit ${ }^{2}$, Noe Montes-Garcia ${ }^{4}$ and \\ Clint W. Magill ${ }^{2}$ \\ ${ }^{I}$ USDA-ARS, Southern Plains Agricultural Research Center, College Station, Texas, USA \\ ${ }^{2}$ Department of Plant Pathology and Microbiology, Texas A\&M University, College Station, Texas 77843, USA \\ ${ }^{3}$ USDA-ARS, Tropical Agriculture Research Station, Mayaguez, Puerto Rico 00680-5470, USA \\ ${ }^{4}$ Instituto Nacional de Investigacuones Forestales, Agricolas y Pecuarias, Centro de Investigación Regional del Noreste, \\ Rio Bravo, Tamaulipas, CP 88900, Mexico
}

\begin{abstract}
Globally, the foliar phase of anthracnose is one of the most destructive diseases of sorghum. In most cases, anthracnose resistance screening relies on the use of a spore suspension. This method is usually conducted after sundown and when there is the possibility of dew formation the following morning. Using a spore suspension for sorghum anthracnose field evaluation in College Station, Texas over five years (1996, 1997, 1999-2001) yielded inconsistent linkage results and failed to identify any closely linked molecular markers. For large scale screening of sorghum germplasm for anthracnose (Colletotrichum sublineolum) resistance, plants are inoculated in the field or in the green house at either $30 \mathrm{~d}$ after planting or at the 8-10 leaf-stage. In field inoculation, the use of C. sublineolum-colonized sorghum grains was shown to be the most efficient and effective in identifying resistant sources. For effective, efficient, fast and accurate infection, approximately 10-20 seeds are placed in each plant leaf whorl and it takes about $16.7 \mathrm{~kg}$ of colonized grains to cover a 0.4 ha area. In the greenhouse, though colonized grains are equally effective, spray inoculation is preferred for easy and uniform coverage. Using this method of inoculum preparation, spore suspension was extracted and sprayed $\left(10^{6}\right.$ conidia $\left.^{-1} \mathrm{l}^{-1}\right)$, followed by $10 \mathrm{hr} / \mathrm{d}$ misting for $30 \mathrm{sec}$ at $30-45 \mathrm{~min}$ interval continuously for a period of one month resulted in effective infection.
\end{abstract}

Keywords: Sorghum, anthracnose, inoculation.

\section{INTRODUCTION}

Anthracnose caused by the fungus Colletotrichum sublineolum P. Henn., in Kabat. and Bubák (syn. C. graminicola (Ces.) G. W. Wilson [1, 2], is one of the most damaging diseases of sorghum [3-5]. Estimating grain yield losses due to anthracnose can often be difficult [6], but losses as high as $50 \%$ have been reported in susceptible cultivars [7-9]. Foliar infection can occur at any stage of plant development, but symptoms are generally observed 40 days after seedling emergence. Characteristic symptoms on susceptible cultivars include small circular to elliptical spots or elongated lesions and as the fungus sporulates, fruiting bodies (acervuli) appear as black spots in the center of the lesions [10]. For identifying disease resistant sources in a large scale germplasm evaluation, it is imperative to identify and follow an effective and efficient inoculation method, both in the field and under green house conditions. To address this problem, an alternate inoculation system which has proven to be extremely effective [11] was used. In this study, C. sublineolum-colonized sorghum grains were used to evaluate sorghum exotic germplasm accessions and

*Address correspondence to this author at the The USDA-ARS, Southern Plains Agricultural Research Center, Crop Germplasm Research, 2881 F\&B Road, College Station, Texas, TX 77845, USA;

E-mail: louis.prom@ars.usda.gov advanced breeding lines in the field. In addition, a total of 320 germplasm accessions received from ICRISAT, India were evaluated under green house condition by using the method of inoculum preparation, spore suspension extraction and spraying to identify anthracnose resistance sources.

\section{MATERIALS AND METHODS}

\section{Inoculum Preparation}

Single spore cultures of Colletotrichum .sublineolum on potato dextrose agar plates, method of large scale inoculum preparation using sorghum grain and microscopic view of C. sublineolum conidia are detailed in Fig. (1).

\section{Field Evaluation}

A total of 100 advanced breeding lines in set I, and 76 exotic (17 from Uganda, 25 from Sudan, 13 from Mali, 15 from Ethiopia and 6 from India) sorghum germplasm lines in set II were evaluated in replicated trials along with BTx623 (susceptible check), and SC748-5 (resistant check) at the research farm, College Station, Texas in 2007. Materials used for field evaluation and method of colonized grains application for anthracnose disease screening is given in Fig. (2).

\section{Green House Evaluation}

A total of 302 (245 - mini-core, 35- high biomass sweet stalk, 15- grain mold tolerant cultivars, 3- high biomass 

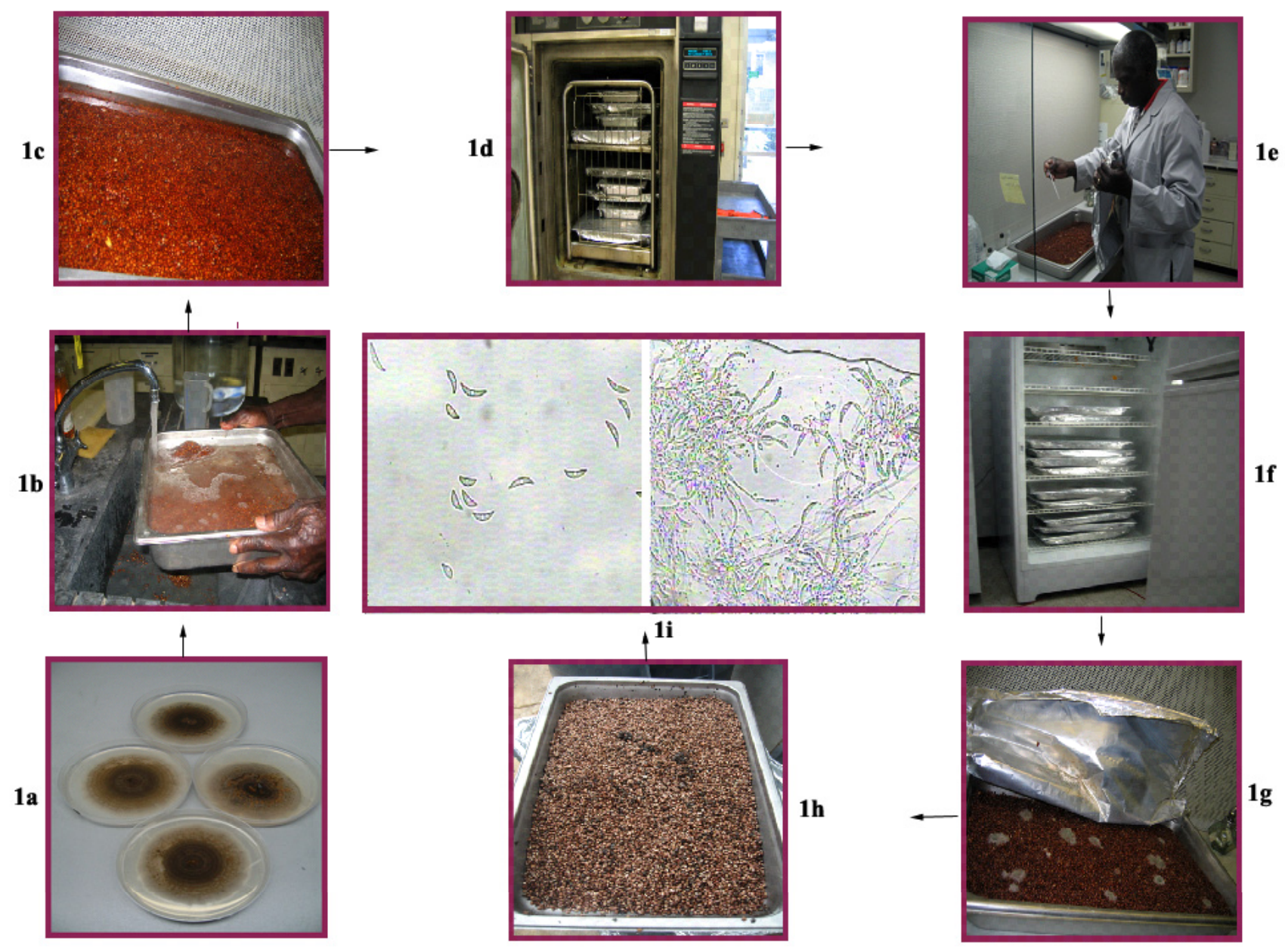

Fig. (1). Colletotrichum sublineolum - Large scale inoculum preparation. (a) Single spore isolates of Colletotrichum sublineolum grown at $25^{\circ} \mathrm{C}$ on half-strength potato dextrose agar. $(\mathbf{b}, \mathbf{c})$ Sorghum grains in stainless steel pans are washed thoroughly under tap water and then soaked for $48 \mathrm{~h}$. (d) After $48 \mathrm{~h}$, the water is drained and the pans are covered with aluminum foil. The pans are autoclaved at $121^{\circ} \mathrm{C}$ for 30 min on two consecutive days. (e) Conidial suspensions or agar plugs containing the fungal isolates are obtained from two-week old culture plates. Under the hood, several drops (10-12) of the conidial suspension or agar plugs containing the fungal isolates are placed on the sterilized sorghum grains and then covered with aluminum foil. (f) Pans containing the mixture of sterilized sorghum grains and fungal isolates are incubated at $25^{\circ} \mathrm{C}$ for $14-21 \mathrm{~d}$. (g) To ensure complete colonization, the inoculated grains are mixed every 3 to $4 \mathrm{~d}$ either by using a sterile spatula under the hood or by shaking the pans without opening the aluminum foil. (h) Colonized sorghum grains are stored in the cold room until used. (i) C. sublineolum conidia as viewed under microscope.

forage and 4-ergot tolerant types) germplasm sorghum lines received from ICRISAT, India along with 16 differentials and BTx623 (susceptible check), and SC748-5 (resistant check) were evaluated two times consecutively in September 2007 and January 2008 in the USDA-ARS green house, College Station, Texas. Detailed method of spray inoculation followed by misting in the green house with photographic illustrations are presented in Fig. (3).

Disease assessments are conducted $30 \mathrm{~d}$ post-inoculation and thereafter on a weekly basis for four consecutive weeks until flowering (Fig. 4). Scoring multiple times enhanced the probability of detecting sporulating lesions. The difference between a resistant and susceptible response is the presence of acervuli on the leaves, which indicates successful reproduction of the pathogen. Ratings are based on a scale of 1-to-5 modified from Pande et al. [13] by Erpelding and Prom [14]. Thakur and Mathur [15] followed 1-5 severity rating scale and suggested a 1-9 severity rating scale for differentiating between lines with minor differences in resistance.

\section{RESULTS AND DISCUSSION}

In a detailed examination of disease development by sorghum anthracnose, Wharton et al. [16] observed that early infection processes (spore germination, differentiation of appresoria, and penetration of sorghum leaf sheaths epidermal cells) were the same in compatible and incompatible interactions. Host responses in both resistant and susceptible cultivars lead to the accumulation of pigments around the sites of infection. These conditions tend to cause mis-scoring of plants, with escapes being assigned a resistant rating. Pande et al. [13] inoculated both leaf surfaces of the plant with a conidial suspension of $C$. sublineolum using a hand sprayer. One hour after inoculation humidifiers were run continuously for $18 \mathrm{hr}$ to create $100 \%$ humidity. Further to promote disease development humidifiers were run continuously for 6 days for $8 \mathrm{hr} /$ day. 


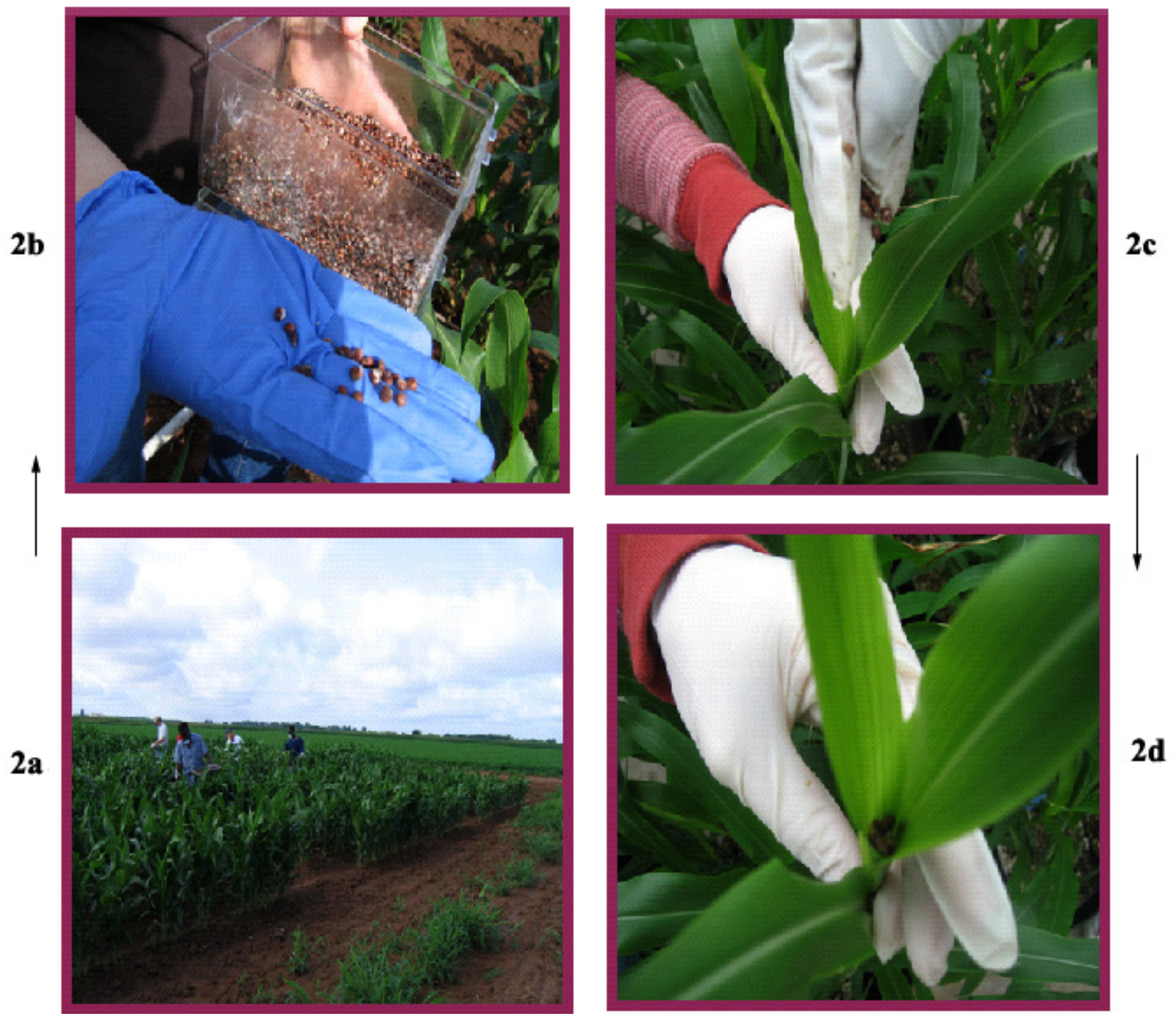

Fig. (2). Field inoculation. (a) Two sets of experiments, one with 100 advanced breeding lines and another with 76 exotic germplasm accessions along with BTx623 (susceptible check), and SC748-5 (resistant check) were evaluated at the College Station Research Farm in 2007 under randomized block design with 3 replicates. Field inoculations are made during the transition from growth stage 1 to growth stage 2 [12], when approximately 8 to 10 leaves are fully developed. (b) C. sublineolum colonized sorghum grains (approximately 10-20 seeds) are placed in each plant leaf whorl. (c) Colonized grains placed in the leaf whorl infect foliar tissues reducing the amount of photosynthate accumulation. Infection of the stalk leads to lodging, a detriment to maximizing harvestable biomass. (d) Sorghum plants are being inoculated with the colonized grains. It takes approx. $16.7 \mathrm{~kg}$ of colonized grains to inoculate a 0.4 ha area.

To confirm disease infection, the author conducted the experiments five times. Mehta [17, 18] followed the same inoculation method by spraying approximately 3-5 $\mathrm{mL}$ of a conidial suspension $\left(10^{6}\right.$ conidia $\left.\mathrm{ml}^{-1}\right)$ onto the leaves and the whorl of each plant, using backpack sprayers or a tractormount sprayer at night or in the early morning so as to have low light intensity. Based solely on anthracnose field scores obtained in this manner (College Station, 1996, 1997, 1999, 2000 and 2001), the author was not able to identify closely linked molecular markers, due to inconsistent phenotyping results for anthracnose resistance from the segregating $\mathrm{F}_{2}$ progeny of the cross BTx623*SC748-5. Mehta [17] reported that the degree of infection on susceptible plants within susceptible or segregating $\mathrm{F}_{2: 3}$ lines were very low compared to the infection observed on susceptible checks indicating a lower level of viable inoculum throughout the evaluated field plots. This made accurate scoring difficult.

To alleviate this problem, an alternate inoculation system, as detailed above, has proven to be extremely effective. Its use here and in the field greatly enhanced the ability to differentiate resistant, segregating and susceptible families [11]. This reclassification of the mapping population clearly revealed the mis-classification in earlier disease phenotyping of the cross BTx623*SC748-5, and also permitted the identification of molecular markers that cosegregate with the anthracnose resistance gene [19]. In a 2007 field evaluation at College Station, Texas, 95\% of the 


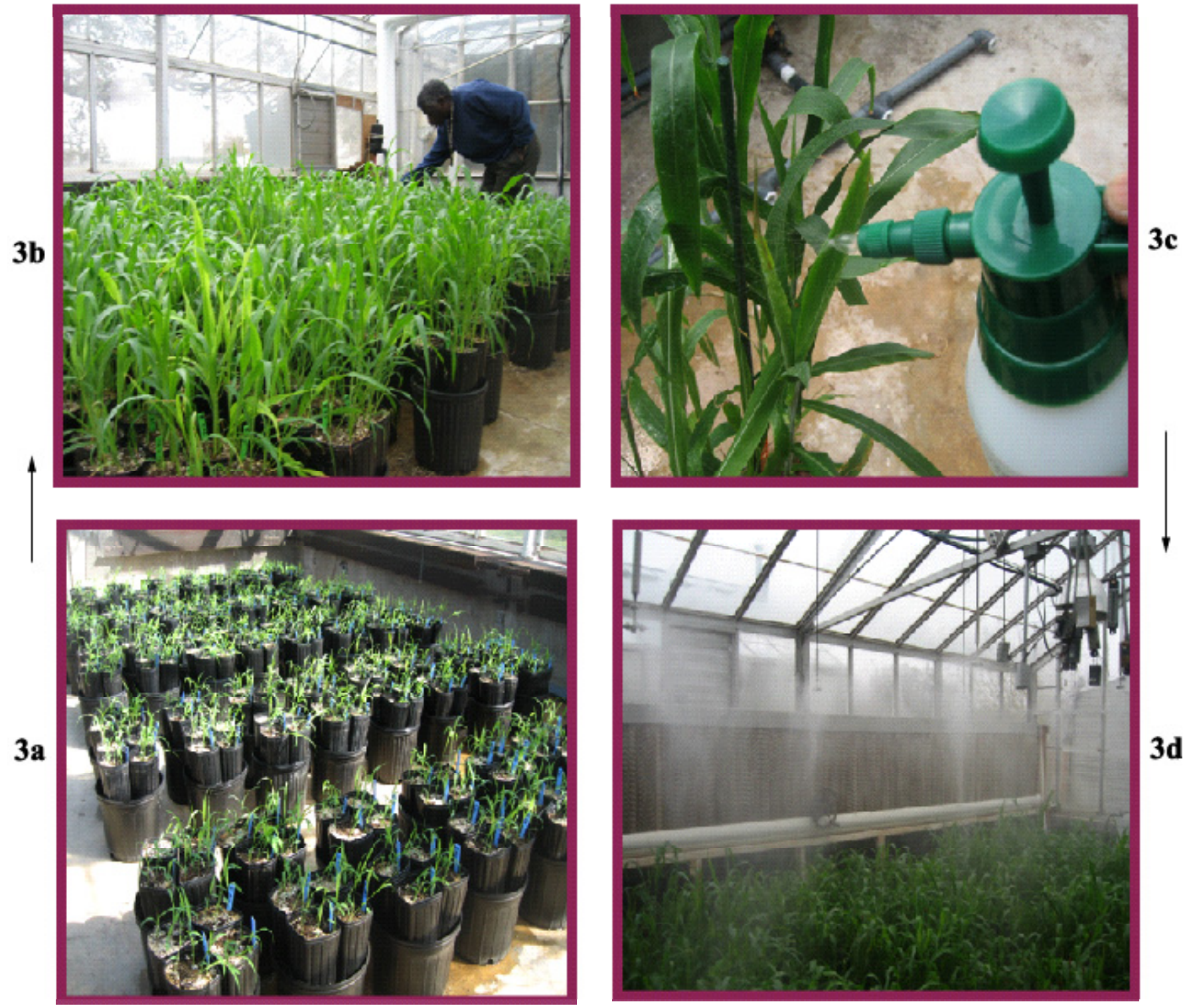

Fig. (3). Green house inoculation. (a) A total of 320 sorghum germplasm lines were evaluated consecutively, in September 2007 and January 2008, along with BTx623 as susceptible and SC748-5 resistant checks under a randomized block design replicated four times. For easy handling, four germplasm accessions, each with five plants, were accommodated in a five gallon pot. (b) Either colonized grains or spray inoculation have been used in the greenhouse to identify new resistance sources with limited spacing between plants and pots in the green house, plant growth won't be as vigorous when compared to field conditions, and makes difficult the placement of colonized grains on individual plant whorls. Hence, for easy and uniform coverage, spray inoculation is preferred for green house screening, though colonized grains application is equally effective. (c) The protocol for colonized grains inoculation is similar to that of the field with the spray inoculation technique, approx. 3-5 $\mathrm{ml}$ conidial suspension $\left(10^{6}\right.$ conidia $\left.\cdot \mathrm{mL}^{-1}\right)$ is deposited on the leaves of each plant. Tween 20 (wetting agent) is added to the inoculum $(0.5 \mathrm{ml} / \mathrm{L})$. (d) Shortly after spraying the inoculum, plants are misted for 30 sec at $30-45 \mathrm{~min}$ intervals, 10 $\mathrm{hr} / \mathrm{d}$ for one month. This misting regime provides favorable environment for infection and disease development.

accessions from 100 advanced germplasm lines and 55\% from the exotic lines were found to be susceptible indicating the effectiveness of the alternate inoculation method developed by Erpelding and Prom [11].

Upadhyaya et al. [20] developed a minicore sorghum germplam (242 accessions from 57 countries) which was representative of entire 2246 germplam collection $(10 \%$ of core, $1 \%$ of entire collection) being maintained as Genetic Resources at International Crops Research Institute for the
Semi-Arid Tropics (ICRISAT), India. Based on many statistical analyses, mini-core germplasm captured about $90 \%$ of the diversity and, majority of the co-adapted gene complexes present in the core. Due to its greatly reduced size and its representation of the entire diversity, the sorghum mini-core was economically evaluated extensively under green house conditions for anthracnose resistance two consecutive times (September 2007 and January 2008). From the screening results, 200 accessions were identified as resistant, 120 accessions were identified as susceptible 

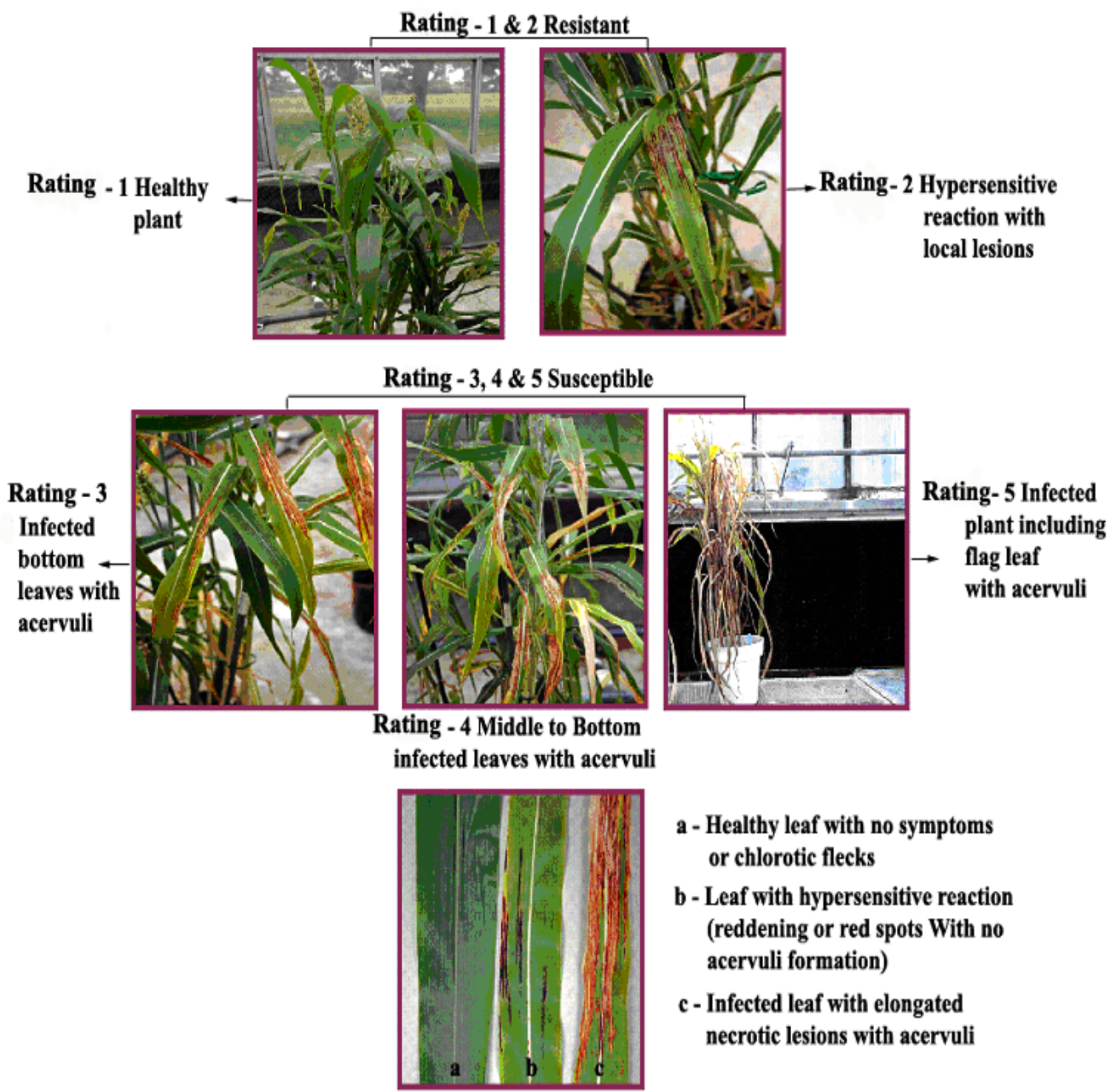

a - Healthy leaf with no symptoms or chlorotic flecks

b - Leaf with hypersensitive reaction (reddening or red spots With no acervuli formation)

c - Infected leaf with elongated necrotic lesions with acervuli

Fig. (4). Disease ratings.

(foliar infection with acervuli), and 11 accessions were identified as susceptible to mid-rib infection. Using the colonized sorghum grain inoculation method, the identified resistance sources will be further evaluated under field conditions in different locations to confirm the stability of this resistance performance and for use in further breeding programs.

\section{REFERENCES}

[1] Sheriff C, Whelan MJ, Arnold GM, Bailey JA. rDNA sequence analysis confirms the distinction between Colletotrichum graminicola and C. sublineolum. Mycol Res 1995; 99: 475-78.

[2] Sutton BC. The Coelomycetes. Commonwealth Mycological Institute, Kew, UK 1980; p. 696.

[3] Ali MEK, Warren IIL. Anthracnose of sorghum. In: de Milliano WAJ, Frederiksen RA, Bengston GD, Eds. Sorghum and Millets Diseases, A Second World Review. ICRISAT, Patancheru, AP, India 1992; pp. 203-208.
[4] Leslie J. Sorghum and Millets Diseases. Iowa St. Press 2003; pp. 63-74.

[5] Pastor-Corrales MA. Variation in pathogenicity of Colletotrichum graminicola (Cesati) Wilson and in symptom expression of anthracnose of Sorghum bicolor (L.) Moench. Ph.D. dissertation. 1980; Texas A\&M University, College Station, TX 1980.

[6] Ngugi HK, Julian AM, King SB, Peacocke BJ. Epidemiology of sorghum anthracnose (Colletotrichum sublineolum) and leaf blight (Exserohilum turcicum) in Kenya. Plant Pathol 2000; 49:129-40.

[7] Harris HB, Johnson BJ, Dobson Jr JW, Luttrell ES. Evaluation of anthracnose on grain sorghum. Crop Sci 1964; 4: 460-2.

[8] Powell P, Ellis M, Alaeda M, Sotomayer AM. Effect of natural anthracnose epiphytotic on yield, grain quality, seed health and seed borne fungi in Sorghum Bicolor. Sorghum News 1977; 20: 7778.

[9] Thomas MD, Sissoko I, Sacco M. Development of leaf anthracnose and its effect on yield and grain weight of sorghum in West Africa. Plant Dis 1995; 80: 151-3.

[10] Thakur RP, Mathur K. Anthracnose. In: Frederiksen RA, Odvody GN, Eds. Compendium of Sorghum Diseases, The American Phytopathological Society, St. Paul, Minneapolis, MN 2000. 
[11] Erpelding JE, Prom LK. Variation for anthracnose resistance within the sorghum germplasm collection from Mozambique, Africa. Plant Pathol J 2006; 5: 28-34.

[12] Pauli AW, Stickler FC, Lawless JR. Developmental phases of grain sorghum as influenced by variety, location and planting date. Crop Sci 1964; 4: 10-13.

[13] Pande S, Mughogho LK, Bandyopadhyay R, Karunakar RI. Variation in pathogenicity and cultural characteristics of sorghum isolates of Colletotrichum graminicola in India. Plant Dis 1991; 75: 778-83.

[14] Erpelding JE, Prom LK. Evaluation of malian sorghum germplasm for resistance against anthracnose. Plant Pathol J 2004; 3 (2): 65 71.

[15] Thakur RP, Mathur K. Anthracnose. In: Thakur RP, Reddy BVS, Mathur K, Eds. Screening Techniques for Sorghum Diseases. ICRISAT, Patancheru, AP, India. Information Bulletin \#76, 2007; pp. 15-23.
[16] Wharton PS, Julian AM, O'Connell RJ. Ultrastructure of the Infection of Sorghum bicolor by Colletotrichum sublineolum. Phytopathology 2001; 91(3): 149-57.

[17] Mehta PJ. Genetic Anthracnose resistance genes in sorghum: characterization and molecular markers. Ph.D. dissertation. Texas A\&M University, College Station, TX 2002.

[18] Mehta PJ, Wiltse CC, Rooney WL, et al. Classification and inheritance of genetic resistance to anthracnose in sorghum. Field Crops Res 2005; 93: 1-9.

[19] Perumal R, Menz MA, Mehta PJ, et al. Molecular mapping of $C g l$, a gene for resistance to anthracnose (Colletotrichum sublineolum) in sorghum. Euphytica 2009; 165(3): 597-606 (DOI 10.1007/s106 81-008-9791-5).

[20] Upadhyaya HD, Reddy VG, Gowda LL, Singh S. A Minicore Collection of Sorghum [Sorghum bicolor (L.) Moench] for Enhancing Utilization of Germplasm in Crop Improvement. ASACSSA-SSSA International Annual Meetings, New Orleans, Louisana. November 4-8, 2007.

Received: January 6, 2009

Revised: February 2, 2009

Accepted: February 6, 2009

(C) Prom et al.; Licensee Bentham Open.

This is an open access article licensed under the terms of the Creative Commons Attribution Non-Commercial License (http: //creativecommons.org/licenses/bync/3.0/) which permits unrestricted, non-commercial use, distribution and reproduction in any medium, provided the work is properly cited. 